\title{
Patterns of venous thromboembolism risk in patients with localized colorectal cancer undergoing adjuvant chemotherapy or active surveillance: an observational cohort study
}

Jakob Michael Riedl 1*, Florian Posch', Angelika Bezan', Joanna Szkandera', Maria Anna Smolle1,3, Thomas Winder², Christopher H. Rossmann ${ }^{1}$, Renate Schaberl-Moser ${ }^{1}$, Martin Pichler ${ }^{1,4}$, Michael Stotz ${ }^{1}$, Herbert Stöger ${ }^{1}$ and Armin Gerger ${ }^{1,3}$

\begin{abstract}
Background: Venous thromoboembolism (VTE) is a frequent and burdensome complication of metastatic colorectal cancer (CRC). However, the epidemiology of VTE in patients with localized CRC after surgery in curative intent is incompletely understood. In this single-center observational cohort study, we investigate patterns of VTE risk in localized CRC, and define its relationship with baseline risk factors, adjuvant chemotherapy and CRC recurrence.

Methods: Five-hundred-sixteen patients with stage II/III CRC were included retrospectively at the time of surgery, and followed until the occurrence of VTE, CRC recurrence, or death (median age $=65.1$ years, stage II and III: $n=151$ (29.5\%), $n=361$ (70.5\%); adjCTX: $n=339$ (65.7\%)).

Results: During a median follow-up of 2.7 years, 15 VTEs (2.7\%) and 116 recurrences (22.5\%) occurred, and 46 patients (8.9\%) died. Six-month, 1-year, and 5-year VTE risks were 1.6\%, 2.0\% and 3.2\%, respectively. In competing risk time-toVTE regression, adjCTX was not associated with an increased risk of VTE (Subdistribution hazard ratio $=0.98,95 \%$ Cl:0. 33-2.88, $p=0.97$ ). The occurrence of disease recurrence strongly increased the risk of VTE (Multi-state model: Transition hazard ratio $(\mathrm{THR})=13.03,95 \% \mathrm{Cl}: 4.39-38.74, p<0.0001))$. Conversely, the onset of VTE did not predict for recurrence $(\mathrm{THR}=1.95,95 \% \mathrm{Cl}: 0.62-6.16, p=0.25)$.

Conclusion: VTE risk is very low in localized CRC and does not appear to be increased by adjuvant chemotherapy. Thus, primary thromboprophylaxis is unlikely to result in clinical benefit in this population. The strongest determinant of VTE risk appears to be disease recurrence.
\end{abstract}

Keywords: Venous thromboembolism, Thrombosis, Colorectal cancer, Recurrence, Adjuvant chemotherapy

\footnotetext{
* Correspondence: j.riedl@stud.medunigraz.at

${ }^{1}$ Division of Clinical Oncology; Department of Medicine; Comprehensive

Cancer Center Graz, Medical University of Graz, Auenbruggerplatz 15, 8036

Graz, Austria

Full list of author information is available at the end of the article
} 


\section{Background}

Venous thromboembolism (VTE) is a frequent complication of malignancy and a leading cause of death in patients with cancer [1]. While the risk of VTE varies greatly between different tumor entities, colorectal cancer (CRC) has been described as a high-VTE-risk disease entity [2]. With a pooled incidence of 33 VTE events per 1000 person-years, CRC harbors the second highest risk of VTE among the four most common cancers in the western world [3]. The majority of data on VTE risk in CRC derives from patients with metastatic disease. High tumor burden, antineoplastic therapy, and reduced performance status exacerbate VTE risk in this setting [4-6]. In contrast, patterns of VTE risk in the localized setting of CRC remain ill-defined. Wellestablished risk factors for VTE, such as surgery, radiotherapy and antineoplastic treatment, are highly prevalent in current neoadjuvant or adjuvant treatment concepts for localized CRC $[7,8]$. Understanding the patterns of VTE risk in this patient population may therefore foster the identification of high-VTE-risk-patients who could benefit from primary thromboprophylaxis. A further important epidemiological aspect is that the relationship between VTE, disease recurrence and death has not been conclusively established in localized CRC.

The study aims to define the patterns of VTE risk in localized CRC. The analysis will draw on observational data to estimate the risk of VTE in localized CRC after curative surgery, and define its association with baseline risk factors, adjuvant chemotherapy and disease recurrence.

\section{Methods}

\section{Study population and design}

Adult patients with stage II or III histologically-verified, localized adenocarcinoma of the colon or rectum referred to our Oncology Division between January, 2010 and March, 2015 represented the population of this singlecenter, retrospective cohort study. All patients with UICC stage IV disease were excluded. Further, patients with neuroendocrine tumors/carcinomas were excluded. However, patients on permanent anticoagulation prior surgery (e.g. for stroke prevention in atrial fibrillation) were eligible for inclusion. Baseline and outcome data were collected retrospectively from our prospectively-maintained electronic healthcare database. The primary endpoint of this study was a composite of objectively-confirmed, symptomatic or incidental deep vein thrombosis and/or pulmonary embolism occurring after surgery in curative intent. VTE events that occurred during neoadjuvant therapy or before tumor diagnosis were not counted as a primary outcome event. Secondary endpoints included disease recurrence and death. Disease recurrence was defined as a composite of local recurrence and/or distant metastasis, whatever came first.

\section{Statistical methods}

All statistical analyses were performed using Stata (Windows version 13.0, Stata Corp., Houston, TX, USA) and R (Windows version 3.1.1., R Core Team (2014), The $\mathrm{R}$ Foundation for Statistical Computing, Vienna, Austria). Continuous variables were reported as medians [25th-75th percentile], whereas categorical data were summarized as absolute frequencies and percentages. For comparing means between two or more groups, we used Wilcoxon rank-sum tests and Kruskal-Wallis tests $[9,10]$. The association between two categorical variables was assessed with $\chi^{2}$-tests or Fisher's exact tests, as appropriate [11, 12]. Median follow-up was calculated with the inverse Kaplan-Meier estimator according to Schemper \& Smith [13]. For the estimation of VTE risk and recurrence risk, we implemented competing risk cumulative incidence estimators according to Marubini \& Valsecchi, considering death-from-any-cause as the competing event of interest (Stata routine stcompet) [14]. The 1-Kaplan-Meier estimator was used for calculating the risk of death-from-any-cause [15]. To dissect the temporal associations between recurrence and VTE, VTE and recurrence, and VTE and death, we fitted three unidirectional illness-death models (Schematic representation: Fig. 1). These multi-state analyses were performed in R, using the mstate library [16]. Proportional baseline hazards were specified for transitions \#2 and \#3 (PH models) [17]. To study the impact of VTE time point on mortality, we extended the multi-state models

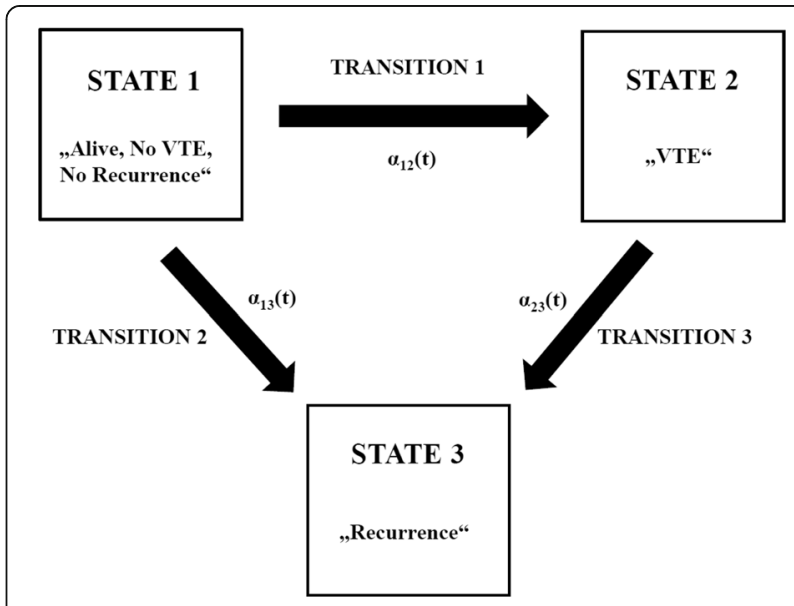

Fig. 1 A unidirectional illness-death model for VTE and recurrence in patients with localized CRC after surgery. The transition hazards for the respective transitions between the states are labeled as $a_{x y}(t)$, respectively. In this three-state, three-transition unidirectional illnessdeath model, the states 1, 2, and 3 represent an initial, transient, and absorbing state, respectively. In state\#1, patients are alive and free from recurrence and VTE after curative surgery. They can either remain in this "initial" state, transit into the "intermediate" state\#2 (transition\#1), or transit into the "absorbing" state\#3 ("recurrence") either directly from state\#1 (transition\#2) or from state\#2 
by including the time-to-VTE as a covariate for transition\#3 (State arrival extended (SAE) model). For multistate based predictions, we generated transition hazards and state occupation probabilities with the msfit and probtrans (implementation of the Aalen estimator) functions of the mstate library [16]. The full analysis code is provided on request from the authors. A general model building framework for multi-state analysis, and relationships with competing risk analysis, are discussed elsewhere [16, 18].

\section{Results}

\section{Analysis at baseline}

Five-hundred-sixteen patients were included in the analysis (Table 1). At baseline, the median age of the cohort was 65.1 years (range 24-91). Approximately half of the cohort suffered from rectal cancer $(n=246$ $(47.9 \%))$, and slightly more than two thirds of patients had stage III disease. Further, two out of three patients were treated with adjuvant chemotherapy (adjCTX) after surgery $(n=339(65.7 \%))$. As compared to patients managed with active surveillance, patients receiving adjCTX were younger (median age: 61.6 vs. 70.4 years, $p<0.0001$ ) more likely to have stage III disease (prevalence of stage III: $79.8 \%$ vs. $55.7 \%, p<0.0001$ ) and had a better performance status (prevalence of patients with a Karnofsky Index $\leq 80 \%$ : $11.1 \%$ vs. $28.9 \%, p<0.0001$ ).

\section{Analysis of event rates}

After a median follow-up interval of 2.7 years (range: 18 days -5.0 years), 15 patients (2.7\%) developed VTE, 116 patients (22.5\%) developed recurrence, and 46 patients (8.9\%) died. Among the 15 VTE events, we observed 10 (66.6\%) DVTs, 4 (26.7\%) PEs, and 1 patient (6.7\%) developed both DVT and PE at the same time. Five venous thrombotic occurrences were not counted as events: Recurrent PE after first in-study PE $(n=1)$, Subclavian vein thrombosis associated with a port-a-cath device $(n=1)$, portal vein thrombosis $(n=2)$, DVT during induction chemotherapy after an insufficient response to neoadjuvant chemoradiation $(n=1)$. Among the 116 recurrences, $13(11.2 \%)$ were local recurrences, 98 (84.5\%) were distant metastasis, and 5 patients (4.3\%) suffered from concurrently detected local recurrence and distant metastasis. In competing risk analysis, the cumulative 6-month, 1-year, and 5-year incidence of VTE was $1.6 \%$ (95\% CI: $0.7-3.0), 2.0 \%(1.0-3.5)$, and $3.2 \%$ (1.9-5.1), respectively. The corresponding risks of recurrence were $5.7 \%(3.9-8.0), 13.1 \%(10.3-16.3)$, and $28.6 \%$ (23.6-33.8), respectively.

\section{Predictors of VTE}

Among the variables reported at baseline, only BMI did significantly differ between patients that did and did not develop VTE during follow-up, with a higher baseline BMI reported in patients developing VTE $(p=0.005$, Table 1). In univariable time-to-VTE competing risk regression (Table 2), a higher BMI emerged as a significant predictor of an increased risk of VTE (Subdistribution hazard ratio (SHR) per $5 \mathrm{~kg} / \mathrm{m}^{2}$ increase in $\mathrm{BMI}=1.57$, 95\% CI: $1.23-2.02, p<0.0001)$. Importantly, adjCTX was not associated with a higher risk of VTE (SHR $=0.98$, 95\% CI: $0.33-2.88, p=0.97)$. In detail, the 6-month, 1 year, and 5-year risks of VTE were 2.1\%, 2.4\% and 3.1\% in the adjCTX group, and $0.6 \%, 1.2 \%$ and $3.6 \%$ in the active surveillance group (Gray's test $\mathrm{p}=$, Fig. 2). As the clinical profile of patients with adjCTX significantly differed from patients undergoing active surveillance, we performed an inverse probability of treatment waited (IPTW) analysis including the variables age, BMI, stage, $\mathrm{T}$ of TNM, N of TNM, Karnofsky index and smoking status. Also here, we did not observe an association between adjCTX and venous thromboembolic events (waited SHR $=0.47,95 \%$ CI: 0.09-2.34, $p=0.35$ ).

\section{Relationship between VTE, recurrence, and death}

In contingency analysis, VTE and recurrence were highly associated with each other (Chi-Squared $p<0.001$ ). In multistate analysis, the onset of recurrence was associated with a 13-fold increase in the risk of VTE $(\mathrm{THR}=13.03,95 \%$ CI: 4.39-38.74, $p<0.0001)$. This finding prevailed after adjusting for BMI $(\mathrm{THR}=12.36$, 95\% CI: 3.32-46.06, $p<0.001)$. In contrast, we did not observe an association between VTE occurrence and a higher risk of cancer recurrence $(\mathrm{THR}=1.95,95 \% \mathrm{CI}$ : $0.62-6.16, p=0.25)$. Recurrences lead to an 18-fold increase in the risk of death (transition hazard ratio $(\mathrm{THR})=18.37,95 \% \mathrm{CI}=9.12-37.00, p<0.0001)$, whereas the onset of VTE was only a weak predictor of an increased risk of death $(\mathrm{THR}=2.76, \mathrm{CI}=0.85-8.95$, $p=0.09$ ).

\section{Discussion}

In this study, we aimed to define patterns of VTE risk in patients with localized colorectal cancer after curative surgery. Overall, we found a very low risk of VTE in the total cohort as well as in the patients who underwent adjuvant chemotherapy. Importantly, adjuvant chemotherapy did not emerge to be a predictor of an increased risk of VTE in this cohort, which does not support the concept that patients undergoing adjuvant chemotherapy after curative surgery for colorectal cancer benefit from primary thromboprophylaxis. The strongest determinant of VTE risk was disease recurrence. (Clinical practis points summarized in Table 3).

Cancer is a major risk factor for VTE [19-21]. Although the risk of cancer-associated VTE strongly varies between tumor types, metastatic CRC is considered 
Table 1 Baseline characteristics of the study population

\begin{tabular}{|c|c|c|c|c|c|}
\hline Variable & $\mathrm{n}$ (\%miss.) & Overall $(n=516)$ & No VTE $(n=501)$ & $\operatorname{VTE}(n=15)$ & $p^{*}$ \\
\hline Age at diagnosis (years) & $516(0.0 \%)$ & $65.1[55.3-72.3]$ & $65.2[55.2-72.4]$ & $62.1[57.0-67.1]$ & 0.28 \\
\hline BMI $\left(\mathrm{kg} / \mathrm{m}^{2}\right)$ & $413(20.0 \%)$ & $25.5[23.0-28.7]$ & $25.4[22.8-28.7]$ & $29.9[25.6-36.6]$ & 0.005 \\
\hline Karnofsky Index at diagnosis (\%) & $347(32.8 \%)$ & / & / & / & 0.23 \\
\hline$\leq 80 \%$ & / & $57(16.4 \%)$ & $56(16.6 \%)$ & $1(11.1 \%)$ & / \\
\hline $90 \%$ & / & $116(33.4 \%)$ & 115 (34.0\%) & $1(11.1 \%)$ & / \\
\hline $100 \%$ & / & $174(50.1 \%)$ & $167(49.4 \%)$ & $7(77.8 \%)$ & / \\
\hline Family history of CRC & $256(50.4 \%)$ & / & / & / & 0.76 \\
\hline No family history & / & $217(84.8 \%)$ & $211(84.7 \%)$ & $6(85.7 \%)$ & / \\
\hline $1^{\text {st }}$ degree relative & / & $25(9.8 \%)$ & $24(9.6 \%)$ & $1(14.3 \%)$ & / \\
\hline $2^{\text {nd }}$ degree relative & / & $14(5.5 \%)$ & $14(5.6 \%)$ & $0(0.0 \%)$ & / \\
\hline Smoker or Ex-Smoker & $358(30.6 \%)$ & $121(33.8 \%)$ & 117 (33.5\%) & $4(44.4 \%)$ & 0.49 \\
\hline Tumor localization & $514(0.4 \%)$ & / & / & / & 0.84 \\
\hline Cecum/Appendix & / & $56(10.9 \%)$ & $56(11.2 \%)$ & $0(0.0 \%)$ & / \\
\hline Ascending colon & / & $37(7.2 \%)$ & $35(7.0 \%)$ & $2(13.3 \%)$ & / \\
\hline Right flexure & / & 15 (2.9\%) & $14(2.8 \%)$ & $1(6.7 \%)$ & / \\
\hline Transverse colon & / & 19 (3.7\%) & $18(3.6 \%)$ & $1(6.7 \%)$ & / \\
\hline Left flexure & / & $22(4.3 \%)$ & $21(4.2 \%)$ & $1(6.7 \%)$ & / \\
\hline Descending colon & / & $9(1.75 \%)$ & $9(1.8 \%)$ & $0(0.0 \%)$ & / \\
\hline Sigma & / & 107 (20.8\%) & 104 (20.8\%) & $3(20.0 \%)$ & / \\
\hline Rectum & / & $246(47.9 \%)$ & 239 (47.9\%) & $7(46.7 \%)$ & / \\
\hline$\geq 2$ localizations & / & $3(0.6 \%)$ & $3(0.6 \%)$ & $0(0.0 \%)$ & / \\
\hline TNM & $509(1.4 \%)$ & / & / & / & 0.75 \\
\hline $\mathrm{T} 1$ & / & $9(1.8 \%)$ & $9(1.8 \%)$ & $0(0.0 \%)$ & / \\
\hline $\mathrm{T} 2$ & / & $27(5.3 \%)$ & $27(5.5 \%)$ & $0(0.0 \%)$ & / \\
\hline T3 & / & $372(73.1 \%)$ & $360(72.9 \%)$ & $12(80.0 \%)$ & / \\
\hline T4 & / & $101(19.8 \%)$ & 98 (19.8\%) & $3(20.0 \%)$ & / \\
\hline TNM & $503(2.5 \%)$ & / & / & / & 0.39 \\
\hline NO & / & 149 (29.6\%) & $144(29.5 \%)$ & $5(33.3 \%)$ & / \\
\hline N1 & / & $215(42.7 \%)$ & $211(43.2 \%)$ & $4(26.7 \%)$ & / \\
\hline $\mathrm{N} 2$ & / & $139(27.6 \%)$ & $133(27.3 \%)$ & $6(40.0 \%)$ & / \\
\hline Stage & $512(0.8 \%)$ & / & / & / & 0.81 \\
\hline Stage II & / & $151(29.5 \%)$ & 147 (29.6\%) & $4(26.7 \%)$ & / \\
\hline Stage III & / & $361(70.5 \%)$ & $350(70.4 \%)$ & $11(73.3 \%)$ & / \\
\hline Adjuvant chemotherapy & $516(0.0 \%)$ & $339(65.7 \%)$ & $329(65.7 \%)$ & $10(66.7 \%)$ & 0.94 \\
\hline
\end{tabular}

Distribution overall and by total recurrence status. Continuous variables are summarized as medians [25th percentile (Q1) - 75th percentile (Q3)], whereas categorical variables are reported as absolute frequencies and percentages. * $p$-values for difference between non-VTE and VTE group are from Pearson's chi-squared tests (categorical variables with expected cell counts $\geq 5$ ), Fisher's exact tests (categorical variables with expected cell counts $<5$ ), or Wilcoxon rank-sum tests (continuous variables)

Abbreviations: $K I$ - Karnofsky Index, TNM - Tumor Node Metastasis classification, BMI - Body mass index, CRC - Colorectal cancer

to be a high-VTE-risk tumor entity, with up to $15 \%$ of patients developing VTE during their course of illness [22]. The risk of VTE in patients with metastatic CRC is highest during the first 6 months after diagnosis, and appears to be further increased by chemotherapy [23-25]. Whether patients with $\mathrm{mCRC}$ would benefit from primary thromboprophylaxis is therefore an important area of ongoing research [26]. In the present study including patients with localized CRC, we observed a very different pattern of VTE risk. First, we observed that the overall risk of VTE for up to 5 years after surgery was as low as $3.2 \%$. Around half of the 15 VTE events observed in this study occurred during the first six months after surgery. This suggests that the post-surgical period is a 
Table 2 Uni- and multivariable predictors of VTE risk in localized CRC

\begin{tabular}{|c|c|c|c|c|c|c|}
\hline \multirow[b]{2}{*}{ Variable } & \multicolumn{3}{|l|}{ Univariable analysis } & \multicolumn{3}{|c|}{ Multivariable analysis adjusting for BMI } \\
\hline & Subdistribution hazard ratio (SHR) & $95 \% \mathrm{Cl}$ & $p$ & Subdistribution hazard ratio (SHR) & $95 \% \mathrm{Cl}$ & $p$ \\
\hline Age at diagnosis (per 5 years increase) & 0.93 & $0.81-1.06$ & 0.29 & 0.85 & $0.70-1.02$ & 0.09 \\
\hline BMI (per 5 kg/m² increase) & 1.57 & $1.23-2.02$ & $<0.0001$ & N/A & N/A & N/A \\
\hline \multicolumn{7}{|l|}{$\mathrm{Kl}$ at diagnosis } \\
\hline $100 \%$ & Ref. & Ref. & Ref. & Ref. & Ref. & Ref. \\
\hline$<100 \%$ & 0.28 & $0.06-1.35$ & 0.11 & 0.31 & $0.07-1.48$ & 0.14 \\
\hline Family history of CRC & 1.00 & $0.12-8.23$ & 0.99 & 1.19 & $0.14-10.22$ & 0.87 \\
\hline Smoker or Ex-Smoker & 1.64 & $0.44-6.09$ & 0.46 & 1.71 & $0.43-6.85$ & 0.45 \\
\hline \multicolumn{7}{|l|}{ Tumor localization } \\
\hline Non-rectal & Ref. & Ref. & Ref. & Ref. & Ref. & Ref. \\
\hline Rectal & 0.97 & $0.35-2.68$ & 0.95 & 1.74 & $0.5-6.11$ & 0.39 \\
\hline \multicolumn{7}{|l|}{ TNM - T } \\
\hline $\mathrm{T} 1 \& \mathrm{~T} 2 \& \mathrm{~T} 3$ & Ref. & Ref. & Ref. & Ref. & Ref. & Ref. \\
\hline T4 & 0.95 & $0.27-3.36$ & 0.94 & 0.53 & $0.06-4.80$ & 0.57 \\
\hline \multicolumn{7}{|l|}{ TNM - N } \\
\hline No & Ref. & Ref. & Ref. & Ref. & Ref. & Ref. \\
\hline$N 1 \& N 2$ & 0.85 & $0.29-2.46$ & 0.76 & 1.14 & $0.28-4.66$ & 0.86 \\
\hline \multicolumn{7}{|l|}{ Stage } \\
\hline Stage ॥ & Ref. & Ref. & Ref. & Ref. & Ref. & Ref. \\
\hline Stage III & 1.15 & $0.37-3.59$ & 0.81 & 1.75 & $0.40-7.63$ & 0.46 \\
\hline Adjuvant chemotherapy & 0.98 & $0.33-2.88$ & 0.97 & 1.18 & $0.27-5.24$ & 0.23 \\
\hline
\end{tabular}

Abbreviations: SHR Subdistribution hazard ratio, 95\% Cl 95\% confidence interval, KI Karnofsky Index, TNM Tumor Node Metastasis classification, BMI Body mass index, CRC Colorectal cancer

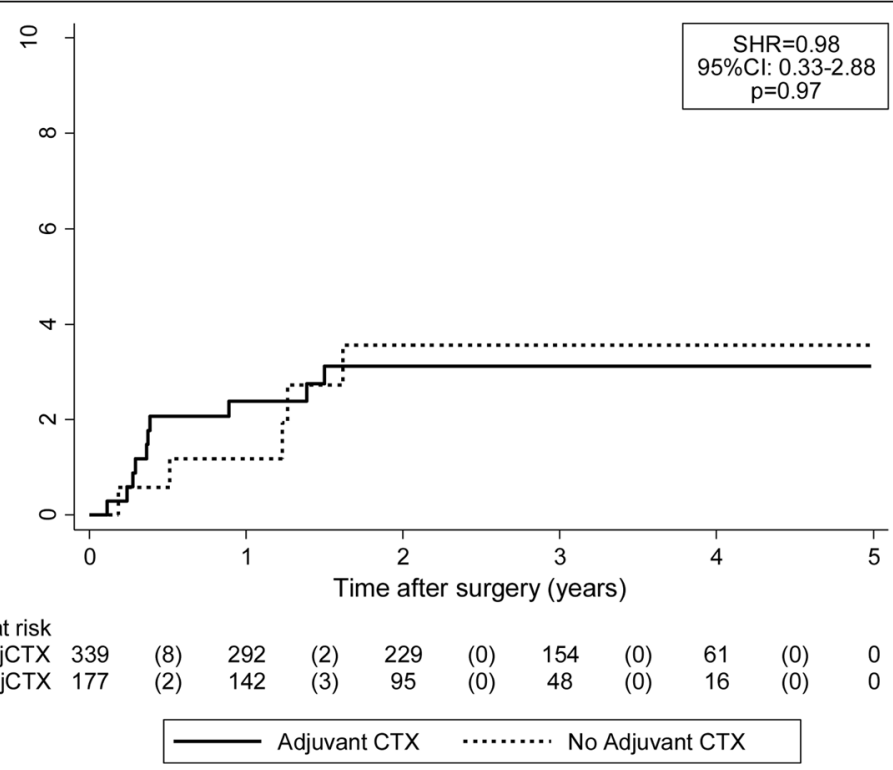

Fig. 2 Cumulative incidence of VTE according to adjuvant chemotherapy status. Note that the $y$-axis scaling only continues until $10 \%$ 
Table 3 Clinical practice points of this study

\begin{tabular}{l}
\hline - In contrast to metastatic colorectal cancer, patients with localized \\
colorectal cancer have a very low risk of VTE during the first three \\
years after surgery in curative intent. \\
- Adjuvant chemotherapy does not appear to increase the risk of VTE. \\
- Primary thromboprophylaxis beyond the recommended extended \\
post-surgical thromboprophylaxis period of 28 days is unlikely to \\
provide clinical benefit in this population. \\
- Disease recurrence after localized colorectal cancer increases the risk \\
of VTE by more than 10 -fold.
\end{tabular}

contributor to VTE risk in CRC patients, and that adherence to extended thromboprophylaxis guidelines in our cohort was high. Interestingly, adjuvant chemotherapy did not emerge to be associated with a higher risk of VTE. This is in contrast to the metastatic setting, where it is believed that chemotherapy is a major contributor towards thrombotic risk. However, chemotherapy is likely not a causal risk factor for cancer-associated VTE, but rather leads to a liberation of prothrombotic agents from cancer cells, including tissue factor bearing microparticles, cell-free DNA, and histones [27, 28].

The low risk of VTE in our cohort of patients with localized disease and the lack of association with adjuvant chemotherapy does not support the hypothesis that patients undergoing adjuvant chemotherapy would benefit from primary thromboprophylaxis for the duration of cytotoxic chemotherapy. Assuming a 50\% relative reduction in the risk of VTE with primary thromboprophylaxis during a 6-month course of adjuvant chemotherapy, this intervention would - according to our 6-month VTE risk of $2.1 \%$ in the adjuvant therapy group - result in a number needed to treat of 125 [29]. Treating 125 patients with a low molecular weight heparin prophylaxis for 6-months to prevent one VTE event would not only incur significant healthcare costs, but also set an excessive number of patients at risk for bleeding complications [30, 31]. Thus, the main message of this study is that primary thromboprophylaxis should not be given to patients with localized CRC after surgery beyond the recommend 28 days of extended post-surgical prophylaxis.

Several predictors for an increased risk of VTE have been identified in patients with metastatic cancers, including - among others - tumor type, lymph node metastasis, elevated D-Dimer, elevated levels of soluble p-selectin, elevated BMI, elevated leukocytes and platelet counts, and anemia/use of erythropoiesis-stimulating agents (ESAs) [32-38]. However, in this study on patients with localized CRC, we could only identify an elevated BMI as an independent predictor of a higher venous thromboembolic risk. A borderline association was observed between advanced age at surgery and a lower risk of VTE. We can speculate that this may be explained by a confounding influence of a more aggressive malignancy in younger patients. In summary, this supports the concept that risk factors for VTE in localized CRC and metastatic CRC may be quite different, and VTE risk prediction tools for the metastatic setting, such as the Khorana score, may not necessarily generalize to patients with localized CRC [36]. Rather, our study suggests that risk factors for VTE in the adjuvant setting of localized CRC are more similar to VTE risk factors in the general population. This notion is particularly supported by the observed strong association of an elevated BMI with VTE in our study.

The temporal relationship between individual endpoints, such as VTE, recurrence, and death can be complex. We therefore implemented multi-state models that allowed us to statistically dissect how VTE and recurrence associate with each other over time, and how they influence the risk of death. Here, we observed that recurrence was a very strong contributor towards VTE risk. The onset of recurrence increased the risk of developing VTE by a factor of more than 13 . This excessive risk increase reflects the transition of patients from the low-VTE-risk localized setting into the high-VTE- recurrent/metastatic setting, and indicates that recurrence is a much stronger risk factor for VTE in localized CRC than any of the baseline variables assessed in this study. Conversely, we only observed a weak and statistically non-significant trend for a relationship between the occurrence of VTE and a higher risk of recurrence. In patients without any evidence of cancer, an "unprovoked" VTE can be an early sign of malignancy [39]. In our study, the absence of a strong association between VTE occurrence and cancer recurrence does not suggest that VTE occurrence may be an early clinical indicator of cancer recurrence. Therefore, we cannot recommend that a VTE event in such a patient should per se lead to a full diagnostic work-up for cancer recurrence. As expected, recurrence emerged as a strong risk factor for death.

Finally, we want to discuss some limitations of this study. First, its retrospective cohort design with retrospective retrieval of thrombotic event data from our in-house electronic healthcare database may have led to an underreporting of VTE events, and thus an underestimation of VTE risk in this study. Further, data on anticoagulation at baseline were not systematically collected, which could have resulted in a "dilution" of VTE risk over time by patients that received continuous anticoagulation for reasons such as atrial fibrillation. Third, within our geographical region, only a fraction of patients suffering from stage I colorectal cancer are referred to our Division of Clinical Oncology. Therefore, we had to exclude stage I patients from this analysis to minimize potential selection bias. However, all patients with stage II and III disease are routinely referred to our 
Division in our region, wherefore our data are generalizable to these patient populations. Fourth, VTE risk may be higher in patients undergoing neoadjuvant chemoradiation for rectal cancer. However, our study does not systematically investigate this treatment period, but rather analyzes VTE risk patterns after surgery. A fifth limitation of our study is that validated biomarkers for cancer-associated VTE in the metastatic CRC setting, such as D-Dimer, were not measured in this study. Moreover, due to the retrospective nature of data ascertainment, we were not able to collect potentially important data on thrombophilia and past history of VTE. This limitation also applies to data on permanent anticoagulation, which were not systematically collected. Permanent anticoagulation, for example to prevent stroke in patients with atrial fibrillation, may have lead to an underestimation of VTE risk in our study. However, given the expected low prevalence of atrial fibrillation in a population with a median age of 65.1 years, we carefully speculate that the magnitude of such a bias will likely be small. Finally, we want to mention two strengths of this study, namely the rigorous adjudication of VTE events, and the application of a statistical methodology ("multi state") that can dissect the complex temporality between VTE, recurrence, and death.

\section{Conclusion}

In this study with a median follow-up of almost 3 years, we have shown that the risk of VTE in patients with localized CRC after curative surgery is very low. Importantly, adjuvant chemotherapy did not emerge as a risk factor for VTE in this setting. Consequently, primary thromboprophylaxis beyond the recommended extended post-surgical thromboprophylaxis is unlikely to provide clinical benefit. The strongest determinant for VTE in this setting is disease recurrence.

\section{Abbreviations \\ adj CTX: Adjuvant chemotherapy; BMI: Body mass index; Cl: Confidence interval; DVT: Deep vein thrombosis; ESAs: Erythropoiesis-stimulating agents; mCRC: Metastatic colorectal cancer; PE: Pulmonary embolism; \\ SHR: Subdistribution hazard ratio; THR: Transition hazard ratio; VTE: Venous thromboembolism}

\section{Acknowledgements}

Not applicable.

\section{Funding}

Funding for personnel costs was provided by SANOFI AVENTIS within an unrestricted research grant to Professor Armin Gerger. SANOFI AVENTIS had no role in the design, conduct, analysis, interpretation, and publication of this study.

\section{Availability of data and materials}

The dataset for this study is not publically available, because datasharing is not approved under the current vote of the local ethic committee in order to protect the anonymity of the patients.

\section{Authors' contributions}

Conceived and designed the study: JMR FP MS AG. Performed statistical analyses: FP Interpreted the results: JMR FP MS JS HS AG. Wrote the first draft of the manuscript: JMR FP. Contributed to the writing of the manuscript: JMR FP AB JS MAS CHR TW RSM MP MS HS AG. Agree with the manuscript's results and conclusions: JMR FP AB JS MAS CHR TW RSM MP MS HS AG. ICMJE criteria for authorship read and met: JMR FP AB JS MAS CHR TW RSM MP MS HS AG. Have read and approved the manuscript: JMR FP AB JS MAS CHR TW RSM MP MS HS AG. All authors read and approved the final manuscript.

\section{Competing interests}

The authors declare that they have no competing interests.

\section{Consent for publication}

Not applicable.

\section{Ethics approval and consent to participate}

The study was approved by the local ethics committee (Ethikkommission der Medizinischen Universität Graz, IRB00002556) prior any patient-related activities were performed (No.25-458 ex 12/13). Written informed consent was not obtained from individual patients, because the local ethics committee specifically granted a "waiver of consent" for this retrospective database study.

\section{Publisher's Note}

Springer Nature remains neutral with regard to jurisdictional claims in published maps and institutional affiliations.

\section{Author details}

${ }^{1}$ Division of Clinical Oncology; Department of Medicine; Comprehensive Cancer Center Graz, Medical University of Graz, Auenbruggerplatz 15, 8036 Graz, Austria. ${ }^{2}$ Department of internal medicine II, LKH Feldkirch, Carinagasse 47, A-6807 Feldkirch, Austria. ${ }^{3}$ Center for Biomarker Research in Medicine, Stiftingtalstrasse 5, $8010 \mathrm{Graz}$, Austria. ${ }^{4}$ Department of experimental therapeutics, The University of Texas MD Anderson Cancer Center, 1901 Eastroad, Houston, TX 77054, USA.

Received: 13 October 2016 Accepted: 26 May 2017

Published online: 15 June 2017

\section{References}

1. Khorana AA, Francis CW, Culakova E, Kuderer NM, Lyman GH. Thromboembolism is a leading cause of death in cancer patients receiving outpatient chemotherapy. J. Thromb. Haemost. England; 2007. p. 632-4

2. Königsbrügge $\mathrm{O}$, Pabinger I, Ay C. Risk factors for venous thromboembolism in cancer : novel findings from the Vienna cancer and thrombosis study (CATS ). Thromb. Res. [Internet]. Elsevier Masson SAS. 2014;133:S39-43. Available from: http://dx.doi.org/10.1016/S0049-3848(14)50007-2

3. Wun T, White RH. Epidemiology of cancer-related venous thromboembolism. Best Pract res Clin Haematol Netherlands. 2009;22:9-23.

4. Chew HK, Davies AM, Wun T, Harvey D, Zhou H, White RH. The incidence of venous thromboembolism among patients with primary lung cancer. J Thromb Haemost England. 2008;6:601-8.

5. Kroger K, Weiland D, Ose C, Neumann N, Weiss S, Hirsch C, et al. Risk factors for venous thromboembolic events in cancer patients. Ann Oncol England. 2006:17:297-303.

6. Numico G, Garrone O, Dongiovanni V, Silvestris N, Colantonio I, Di Costanzo $\mathrm{G}$, et al. Prospective evaluation of major vascular events in patients with nonsmall cell lung carcinoma treated with cisplatin and gemcitabine. Cancer United States. 2005;103:994-9.

7. Labianca R, Nordlinger B, Beretta GD, Mosconi S, Mandala M, Cervantes A, et al. Early colon cancer: ESMO Clinical Practice Guidelines for diagnosis, treatment and follow-up. Ann. Oncol. England; 2013;24 Suppl 6:vi64-vi72.

8. Glimelius B, Tiret E, Cervantes A, Arnold D. Rectal cancer: ESMO Clinical Practice Guidelines for diagnosis, treatment and follow-up. Ann. Oncol. England; 2013;24 Suppl 6:vi81-8.

9. Wilcoxon F. Individual comparisons of grouped data by ranking methods. J. Econ. Entomol. Not Available; 1946;39:269. 
10. Kruskal WH, Wallis WA. Use of Ranks in One-Criterion Variance Analysis. J. Am. Stat. Assoc. [Internet]. [American Statistical Association, Taylor \& Francis, Ltd.]; 1952;47:583-621. Available from: http://www.jstor.org/stable/2280779

11. Yates F. Contingency Tables Involving Small Numbers and the \&\#x3c7; Test. Suppl. to J. R. Stat. Soc. [Internet]. [Wiley, Royal Statistical Society]; 1934;1:217-35. Available from: http://www.jstor.org/stable/2983604.

12. Fisher RA. On the Interpretation of \&\#x3c7; from Contingency Tables, and the Calculation of P. J. R. Stat. Soc. [Internet]. [Wiley, Royal Statistical Society]; 1922;85:87-94. Available from: http://www.jstor.org/stable/2340521.

13. Schemper $M$, Smith TL. A note on quantifying follow-up in studies of failure time. Control Clin Trials UNITED STATES. 1996;17:343-6.

14. Marubini E, Morabito A, Valsecchi MG. Prognostic factors and risk groups: some results given by using an algorithm suitable for censored survival data. Stat med ENGLAND. 1983;2:295-303.

15. Kaplan EL, Meier P. Nonparametric Estimation from Incomplete Observations. J. Am. Stat. Assoc. [Internet]. [American Statistical Association, Taylor \& Francis, Ltd.]; 1958:53:457-81. Available from: http://www.jstor.org/stable/2281868.

16. de Wreede LC, Fiocco $M$, Putter $H$. The mstate package for estimation and prediction in non- and semi-parametric multi-state and competing risks models. Comput Methods Programs Biomed Ireland. 2010;99:261-74.

17. Putter H, Fiocco M, Geskus RB. Tutorial in biostatistics: competing risks and multi-state models. Stat med England. 2007;26:2389-430.

18. Posch F, Riedl J, Reitter E-M, Kaider A, Zielinski C, Pabinger I, et al. Hypercoagulabilty, venous thromboembolism, and death in patients with cancer. A Multi-State Model Thromb Haemost Germany. 2016;115:817-26.

19. Prandoni P, Falanga A, Piccioli A. Cancer and venous thromboembolism. Lancet Oncol England. 2005;6:401-10.

20. Naess IA, Christiansen SC, Romundstad P, Cannegieter SC, Rosendaal FR, Hammerstrom J. Incidence and mortality of venous thrombosis: a population-based study. J. Thromb. Haemost. England. 2007;5:692-9.

21. Heit JA, O'Fallon WM, Petterson TM, Lohse CM, Silverstein MD, Mohr DN, et al. Relative impact of risk factors for deep vein thrombosis and pulmonary embolism: a population-based study. Arch. Intern. Med. United States. 2002;162:1245-8.

22. Chew HK, Wun T, Harvey D, Zhou H, White RH. Incidence of venous thromboembolism and its effect on survival among patients with common cancers. Arch Intern med United States. 2006;166:458-64

23. Alcalay A, Wun T, Khatri V, Chew HK, Harvey D, Zhou H, et al. Venous thromboembolism in patients with colorectal cancer: incidence and effect on survival. J Clin Oncol. 2006;24:1112-8.

24. Blom JW, Doggen CJM, Osanto S, Rosendaal FR. Malignancies, prothrombotic mutations, and the risk of venous thrombosis. JAMA United States. 2005;293:715-22.

25. Vormittag R, Simanek R, Ay C, Dunkler D, Quehenberger P, Marosi C, et al. High factor VIII levels independently predict venous thromboembolism in cancer patients: the cancer and thrombosis study. Arterioscler Thromb Vasc Biol United States. 2009;29:2176-81.

26. Posch F, Konigsbrugge $\mathrm{O}$, Zielinski C, Pabinger I, Ay C. Treatment of venous thromboembolism in patients with cancer: a network meta-analysis comparing efficacy and safety of anticoagulants. Thromb res United States. 2015;136:582-9.

27. Thaler J, Koder S, Kornek G, Pabinger I, Ay C. Microparticle-associated tissue factor activity in patients with metastatic pancreatic cancer and its effect on fibrin clot formation. Transl res United States. 2014;163:145-50.

28. Demers M, Krause DS, Schatzberg D, Martinod K, Voorhees JR, Fuchs TA, et al. Cancers predispose neutrophils to release extracellular DNA traps that contribute to cancer-associated thrombosis. Proc. Natl. Acad. Sci. U. S. a. United States. 2012;109:13076-81.

29. Agnelli G, George DJ, Kakkar AK, Fisher W, Lassen MR, Mismetti P, et a Semuloparin for thromboprophylaxis in patients receiving chemotherapy for cancer. N. Engl. J. Med. United States. 2012;366:601-9.

30. Prandoni P, Lensing AWA, Piccioli A, Bernardi E, Simioni P, Girolami B, et al. Recurrent venous thromboembolism and bleeding complications during anticoagulant treatment in patients with cancer and venous thrombosis. Blood. United States. 2002;100:3484-8.

31. Hutten BA, Prins MH, Gent M, Ginsberg J, Tijssen JG, Buller HR. Incidence of recurrent thromboembolic and bleeding complications among patients with venous thromboembolism in relation to both malignancy and achieved international normalized ratio: a retrospective analysis. J Clin Oncol UNITED STATES. 2000;18:3078-83.
32. Dickmann B, Ahlbrecht J, Ay C, Dunkler D, Thaler J, Scheithauer W, et al. Regional lymph node metastases are a strong risk factor for venous thromboembolism: results from the Vienna cancer and thrombosis study. Haematologica Italy. 2013:98:1309-14.

33. Ay C, Simanek R, Vormittag R, Dunkler D, Alguel G, Koder S, et al. High plasma levels of soluble $\mathrm{P}$-selectin are predictive of venous thromboembolism in cancer patients: results from the Vienna cancer and thrombosis study (CATS). Blood United States. 2008;112:2703-8.

34. Ay C, Vormittag R, Dunkler D, Simanek R, Chiriac A-L, Drach J, et al. D-dimer and prothrombin fragment $1+2$ predict venous thromboembolism in patients with cancer: results from the Vienna cancer and thrombosis study. J Clin Oncol United States. 2009;27:4124-9.

35. Khorana AA, Francis CW, Culakova E, Kuderer NM, Lyman GH. Frequency, risk factors, and trends for venous thromboembolism among hospitalized cancer patients. Cancer. United States. 2007:110:2339-46.

36. Khorana AA, Kuderer NM, Culakova E, Lyman GH, Francis CW. Development and validation of a predictive model for chemotherapy-associated thrombosis. Blood. United States. 2008;111:4902-7.

37. Tonia T, Mettler A, Robert N, Schwarzer G, Seidenfeld J, Weingart O, et al. Erythropoietin or darbepoetin for patients with cancer. Cochrane database Syst. Rev. England; 2012;12:CD003407.

38. Posch F, Thaler J, Zlabinger G-J, Konigsbrugge O, Koder S, Zielinski C, et al. Soluble vascular endothelial growth factor (SVEGF) and the risk of venous thromboembolism in patients with cancer: results from the Vienna cancer and thrombosis study (CATS). Clin Cancer res United States. 2016;22:200-6.

39. Carrier M, Lazo-Langner A, Shivakumar S, Tagalakis V, Zarychanski R, Solymoss $\mathrm{S}$, et al. Screening for occult cancer in unprovoked venous thromboembolism. N Engl J med United States. 2015;373:697-704.

\section{Submit your next manuscript to BioMed Central and we will help you at every step:}

- We accept pre-submission inquiries

- Our selector tool helps you to find the most relevant journal

- We provide round the clock customer support

- Convenient online submission

- Thorough peer review

- Inclusion in PubMed and all major indexing services

- Maximum visibility for your research

Submit your manuscript at www.biomedcentral.com/submit
) Biomed Central 